\title{
Partitioning species and environmental diversity in fragmented landscapes: do the alpha, beta and gamma components match?
}

\author{
Yoni Gavish ${ }^{1,2}$ - Itamar Giladi ${ }^{3} \cdot$ Yaron Ziv $^{2}$
}

Received: 8 June 2018 / Revised: 1 November 2018 / Accepted: 21 December 2018 /

Published online: 4 January 2019

(C) The Author(s) 2019

\begin{abstract}
To understand patterns of alpha, beta and gamma diversities in fragmented landscapes we need to explore the three scale components in relation to potential drivers in a scaledependent manner. Often, the drivers themselves can be partitioned to alpha, beta and gamma diversities. Thus, one can hypothesize that the scale-components of species diversity and drivers' diversity match, i.e., that species alpha diversity is mainly explained by drivers' alpha diversity, beta by beta and gamma by gamma. Here, we explore this 'scalematching' hypothesis for spiders in two fragmented agricultural landscapes. In each landscape, we sampled spiders and their potential prey in 12 patches. Then, we sub-sampled pseudo-landscapes in which we calculated spider alpha, beta and gamma diversities using multiplicative diversity-partitioning. Next, we used variance partitioning analysis to explore the relative contribution of eleven explanatory variables from five thematic groups (sampling intensity, area, connectivity, habitat diversity and prey diversity), while further partitioning the habitat and prey diversities to their corresponding alpha, beta and gamma diversities. We found considerable evidence for scale-matching, with spiders' alpha and beta diversities explained mostly by the corresponding alpha and beta diversities (respectively) of prey and/or habitat. We further found a strong effect of connectivity on spider beta diversity, but not on alpha and gamma diversities. For spiders gamma diversity, a cross-scale effect was observed. Our results suggest that multiple drivers from multiple scales interact in structuring patterns of spider alpha, beta and gamma diversities in agroecosystems, yet the strongest effects are of those drivers that match in scale.
\end{abstract}

Keywords Agroecosystems · Araneae - Community composition · Effective diversity · Fragmentation $\cdot$ Meta-community $\cdot$ Scale

Communicated by Andreas Schuldt.

Electronic supplementary material The online version of this article (https://doi.org/10.1007/s1053 1-018-01691-7) contains supplementary material, which is available to authorized users.

Yoni Gavish

gavishyoni@gmail.com

Extended author information available on the last page of the article 


\section{Introduction}

To protect biodiversity from the undesirable effects of climate and land-use change we need to understand how diversity is distributed, and identify the main drivers affecting the distribution patterns. Species diversity in both natural systems and fragmented landscapes, is affected by multiple processes operating at multiple scales. However, it is difficult to understand the drivers' effects on species diversity without first clarifying the scales at which diversity and its drivers are linked and measured. Whittaker (1972) suggested that species diversity can be described at three scales - within locations (i.e., alpha diversity), between locations (i.e., beta diversity) and over the entire extent (i.e., gamma diversity). Usually, alpha and gamma diversities are quantified directly from the data, while beta diversity is expressed as either the gamma-to-alpha ratio (in the multiplicative approach, Jost 2007, 2010) or the gamma-to-alpha delta (in the additive approach, Crist and Veech 2006; Lande 1996). Alpha, beta and gamma diversities can be measured at different geographic grains and extents, depending on the researchers' choice and the specific study question. Nonetheless, since the three diversity components are related to one another, a better understanding of alpha, beta and gamma diversity patterns could be obtained if they were all explored simultaneously.

Similar to species diversity, many of the potential abiotic and biotic drivers of diversity patterns can themselves be explored at multiple scales. Some drivers can be partitioned to their diversity components in a similar manner to the partitioning of species diversity. For example, habitat diversity may be explored over the entire landscape, at the patch scale or as the turnover of habitats between patches (Giladi et al. 2011; Hortal et al. 2009; Tews et al. 2004; Weibull et al. 2003; Wulf and Kolk 2014). Other drivers cannot be partitioned, but can be quantified at different scales. For example, connectivity can be explored as a property of an entire landscape (Urban and Keitt 2001), as the position and spatial context of specific patches within the landscape (Giladi et al. 2011; Zulka et al. 2014), or as the variance of patch-level connectivity. Likewise, area per-se effects can be explored as the entire cover of natural habitat in the landscape (Hanski et al. 2013), as the area of a single patch (i.e., species-area relationship, Rosenzweig 1995) or as properties of the distribution of area among patches (e.g., area scale, Triantis et al. 2012). Since both species diversities and potential driver diversities are scale-dependent it is important to explore which scale components of diversity are explained by different scale components of the drivers.

Here we focus on a new and simple hypothesis - the scale-matching hypothesis - which states that species alpha, beta and gamma diversities should be mainly affected by their drivers' corresponding scale-components. For example, if both species diversity and habitat diversity can be partitioned into their scale components, scale matching would imply that species alpha diversity is better explained by habitat alpha diversity than by habitat beta or habitat gamma diversity.

The rationale underlying the scale-matching hypothesis is based on strong theoretical considerations. Niche theory posits that each species can survive within a limited niche space, which differs from niche spaces that support other species. Locations with higher alpha diversity of habitats are likely to provide a wider niche space, thus increasing the number of species that can co-exist. Landscapes exhibiting high turnover of habitats between patches are expected to support different communities in each patch, resulting in high species beta diversity. Similar niche-based argument also stand for scale-matching between trophic levels (e.g., predators and prey diversities, herbivores and plants, pollinators and plants). However, scale-matching between trophic levels may also arise under 
neutral dynamics if the number of species in each trophic level is restricted by the total number of individuals of the trophic level (Hubbell 2001). For example, when the total abundance of both predators and prey is higher in certain locations due to similar response to higher overall productivity gradient, scale matching between the two trophic levels may arise without any niche-based processes.

Despite the simplicity of the scale-matching hypothesis, we are not aware of any publication that explored it in full. One potential reason for the scarcity of studies is that its statistical evaluation should ideally be based on sampling of species diversity and drivers components at different scales and across multiple landscapes (Zhang et al. 2014). Studies that sampled multiple landscapes within a single study usually focused on one (Jamoneau et al. 2012; Papanikolaou et al. 2017) or two (Ekroos et al. 2010; Simmering et al. 2006) of the scale components (i.e., either alpha, beta or gamma, or a combination of two of them). When all three scale components have been measured, a limited set of drivers have been considered (Hendrickx et al. 2009; Poggio et al. 2010). Furthermore, none of the manuscripts we found partitioned the diversity of habitats, prey, or other resources to their scalespecific components. Finally, most the above studies relied on species richness, ignoring any variation between species in abundances, biomass or cover. For example, Papanikolaou et al. (2017) explored wild bees and plant diversity in 24 agricultural landscapes, and sampled 79-314 plots per landscape. For species richness, they pooled the data from all the plots in each landscape (i.e., gamma diversity), and found a strong correlation between plant and bee richness. However, they have not explored if the mean per-plot richness of bees and plants match across landscapes (alpha scale) or if within-landscape turnover of bees and plants correlate to one another. In addition, they have not explored cross-scale effects, e.g., is the mean per-plot richness of bees affected by the total gamma diversity of plants?

In fact, scale-matching is usually assumed a priori when selecting variables to focus on. For example, multiple studies at the patch or landscape scales found correlation between insect and plant richness or diversity (Fournier et al. 2017; Kemp and Ellis 2017; Martinez et al. 2015; Papanikolaou et al. 2017), between prey and predator richness and diversity (Sandom et al. 2013; Yang et al. 2018) and between species and habitat diversity (e.g., Hortal et al. 2006). But most of these studies did not explore potential cross-scale effects. Likewise, distance decay patterns are usually explored in relation to difference between sites in environmental conditions. However, they are rarely explored in relation to the mean condition of the pair of sites, which may be quite relevant if the two sites are close enough spatially to be linked by dispersal. For example, Prober et al. (2015) found that plant beta diversity explained beta diversity of soil microbes, and found no relation between plot alpha diversity of plant and soil microbes. However, they have not explored potential crossscale effects. On the contrary, Gossner et al. (2016) found that pairwise beta diversity of multiple taxa is correlated with the pairwise difference between sites in land-use intensification trends (i.e., a beta-scale), but also with the mean land-use intensity of the two sites (i.e., an alpha scale measure).

In addition to scale-matching effects, the diversity of a given driver at a given scale may affect species diversity at a different scale, i.e., a cross-scale effect. If some species require multiple habitats to survive throughout their lifetime (e.g., nesting vs. foraging habitats for birds), higher gamma diversity of habitats will promote alpha diversity of more mobile organisms. However, many cross-scale effects may be mediated by more direct scalematching components. For example, habitat gamma diversity may affect species gamma diversity of habitat specialists, and species gamma diversity may then affect species alpha diversity through mass effects (Leibold et al. 2004; Mouquet and Loreau 2002; Shmida and 
Wilson 1985). Empirical evidence for cross-scale effects is commonly found in studies that explored the effect of the landscape context on patch-level diversity (Fahrig 2013; Malavasi et al. 2016).

Here, we aim to explore the scale-matching hypothesis for spider diversity in two fragmented agricultural landscapes from Israel. Although many species are likely to be adversely affected by fragmentation, fragmented landscapes still have the potential to support a considerable proportion of the natural flora and fauna (Gavish et al. 2012; Mendenhall et al. 2014). We analyzed the three diversity components in relation to multiple explanatory variables from five thematic groups (sampling intensity, area, connectivity, habitat diversity and prey diversity). We further partitioned habitat diversity and prey diversity to their corresponding diversity components. If the scale-matching hypothesis is supported, we predict that spider alpha, beta and gamma diversities would be mainly explained by their corresponding diversity components of habitat and prey diversities. We further predict that all scale components should be affected by variables relating to the sampling and area drivers, yet connectivity (as measured here) will mainly explain spider beta diversity. As we only have 2 landscapes, we used a subsampling approach in which we create multiple 'pseudo-landscapes' for each real landscape and explore the scale-matching hypothesis in these pseudo-landscapes. Although the low number of true replicates may influence our conclusions for this specific case-study, scale-matching is usually assumed a-priory when selecting drivers, and as far as we know, has not been explored (or even properly formulated) before.

\section{Methods}

\section{Study sites and sampling}

We focused our sampling in the fragmented landscapes of the Southern Judea Lowland (SJL), Israel, located at the sharp transition zone between the Mediterranean ecosystem in the north of Israel, and the arid ecosystem in the south. Within SJL, we focused on two $3.2 \times 4 \mathrm{~km}$ (1280 ha) landscapes (Dvir and Lachish), both fragmented by agricultural activity, with wheat as the main crop. Both landscapes contain remnant natural patches of various sizes and shapes, in which we sampled spiders and other invertebrates (as potential prey). Sampling took place during the hot summer months (June-early September 2007). The samples were distributed between the landscapes following a three-day cycle (with one additional landscape that is not included in this analysis). We used aerial photographs with $1 \mathrm{~m}^{2}$ resolution to manually digitize all the natural vegetation patches within each landscape. We then selected 12 patches in each landscape, with patch area ranging from 0.110 to 3.900 ha (mean: 1.099) in Dvir and from 0.060 to 2.808 ha (mean: 0.882) in Lachish (see online resource 1).

We took a total of 158 samples in each landscape and increased our sampling intensity with patch size in three area-based steps (online resource 1). Each patch was visited at least twice during the sampling season. Due to the semi-destructive sampling method (see below) sampling locations were not revisited, and instead nearby new sampling locations were selected each visit. Each sample covered an area of $0.5 \times 0.5 \mathrm{~m}$, and contained a single habitat. The list of 9 predefined habitats contained four structurally simple ones (exposed soil, annual plants $<15 \mathrm{~cm}$ tall, annual plants $\geq 15 \mathrm{~cm}$ tall and rosette plants) and five structurally complex habitats (two of these patch types were defined by the presence of 
a common perennial; either Sarcopoterium spinosum, or Hyparrhenia hirta, and the others by the main vegetation structure, bushes $\geq 35 \mathrm{~cm}$ tall, shrubs $<35 \mathrm{~cm}$ tall and thistles). The simple and complex habitats differ considerably in conditions and in spiders' community structure (Gavish and Ziv 2016).

To obtain a single sample of arthropods (including spiders), we used the vacuum option of a leaf blower, with a $0.5 \mathrm{~mm}$ mesh sleeve covering the suction tube (Stewart and Wright 1995). All the sucked debris was collected and sorted in the lab, where all arthropods larger than $0.5 \mathrm{~mm}$ were extracted using a set of sieves. Spiders were identified to the species or morphospecies level, while all other arthropods (potential prey) were identified to the order or family level (see Gavish et al. 2012 for a more detailed description of the sampling methods). As we were not interested in within-patch species turnover, we pooled all samples within a patch to represent its spider and potential prey local communities, and used these local communities when estimating the mean alpha-diversity over all patches.

In addition, in each patch we took 4-11 line transects to estimate the relative cover of each habitat (online resource 1). The number of transects increased with patch size, for a total of 85 transects per landscape. Starting locations and angles were randomly chosen, ensuring that the entire transect fell within the patch (and not closer than 5 meters from the edge). Each transect was $20 \mathrm{~m}$ long, and we recorded the dominant habitat in 160 cells (each with an area of $0.25 \times 0.25 \mathrm{~m}$ ) located on both sides of the line. We included the nine habitats listed above, with the addition of bare-rock cover (which was not sampled for spiders). We pooled the data from all transects taken within a patch to calculate the relative cover of each habitat in each patch. Transect surveys were conducted during September 2007, immediately following the arthropod sampling.

\section{Data preparation}

Our approach was to use the sampled patches in the two real landscapes to create pseudolandscapes by choosing various sub-samples of the patches. For that purpose, within each of the two landscapes, we listed all possible sub-samples of 3, 4, 5, 6, 7, 8, and 9 patches and analyzed data from each of these sub-samples as a pseudo-landscape. Thus within each real landscape we created totals of 220, 495, 792, 924, 792, 495, and 220 pseudo-landscapes in each of the seven patch-cardinalities (i.e., 3-9 patches respectively).

In each pseudo-landscape we calculated the mean alpha, the beta and the gamma diversities of the dependent variable, spider diversity. As the patches differed in the total number of individuals and sampling intensity, we used the effective diversity based on Shannon diversity (Shannon diversity: $-\sum_{s=1}^{S}\left[P_{s, j} \cdot \ln \left(P_{s, j}\right)\right]$, see notations below), as recommended for unequal weights (when samples differ in their total abundances) (Jost 2007). We have used Shannon's effective diversity (i.e., the exponent of the weighted mean of Shannon diversity) and not species richness since patches can differ considerably in relative abundances despite having similar species composition.

To calculate the effective diversity we first estimated the mean patch-level alpha diversity $\left(D_{\alpha}\right)$ of a pseudo-landscape using:

$$
D_{\alpha}=\exp \left(-\sum_{j=1}^{J}\left(W_{j} \cdot \sum_{s=1}^{S}\left(P_{s, j} \cdot \ln \left(P_{s, j}\right)\right)\right)\right.
$$

with $P_{s, j}$ being the relative abundance of species $s$ in patch $j$, and the weights $W_{j}$ as the total number of individuals in the patch divided by the total number of individuals in all patches. 
Next, we pooled the data from all the patches in the pseudo-landscape to estimate gamma diversity $\left(D_{r}\right)$ :

$$
D_{\gamma}=\exp \left(-\sum_{s=1}^{S}\left(P_{s,+} \cdot \ln \left(P_{s,+}\right)\right)\right)
$$

with $S$ being the total number of species in the pseudo-landscape, and $P_{s,+}$ the relative abundance of species $s$ from the total number of individuals in the pseudo-landscape. For the calculation of beta diversity, we followed the multiplicative diversity partitioning framework based on effective numbers (Jost 2007) and estimated the spider beta diversity $\left(D_{\beta}\right)$ as the ratio of the gamma and alpha diversities:

$$
D_{\beta}=D_{\gamma} / D_{\alpha}
$$

For each pseudo-landscape we calculated values for 11 different explanatory variables relating to sampling intensity (1 variable), area (2), connectivity (2), prey diversity (3), and habitat diversity (3). The sampling intensity variable was defined as the total number of samples taken in all patches together. The first area-related variable was the total area of all patches. The second area-related variable was the "area evenness"- the effective diversity of the area distribution by using Eq. (2) above, with the relative area of each patch as the proportions. Larger values of area evenness indicate a more even distribution of area between the patches. Connectivity was defined as the length of the edge-to-edge minimum spanning tree that connects all the patches in the pseudo-landscape (Urban and Keitt 2001), where larger values indicate less connectivity. This connectivity index is sensitive to the distribution of patches in the landscape, yet it does not account for the area of each patch. Thus, we also calculated a clumping index by dividing the total area of all the patches in the pseudo-landscape by the area of the minimum-convex polygon around all patches, returning larger values if the patches are large relative to the inter-patch distances (i.e., high connectivity). Both connectivity measures are at the gamma scale.

The calculations of alpha, beta and gamma diversities of prey and habitat heterogeneity followed the same procedure as described above for spider diversity with the following substitutions. For prey, we used relative abundance of insect family (or order) instead of species abundance. For habitat diversity, we used the relative cover of each habitat type instead of species abundance with the relative number of transects per patch as the patch weight.

\section{Statistical analysis}

To explore the general dependency of spider diversity on each of the explanatory variables we first fitted a separate least-square linear regression model for each of the spider diversity components (alpha, beta or gamma) as dependent variable against each of the eleven explanatory variables in turn. We repeated this analysis for each of the seven patchcardinalities, separately in each landscape, for a total of 462 linear models (3 dependent variables $\times 11$ explanatory variables $\times 7$ patch-cardinalities $\times 2$ landscapes).

Then, we used multiple linear regressions and hierarchical variance partitioning to explore the relative importance of each of the 11 explanatory variables and to test the scale-matching hypotheses. We repeated the following procedure separately for each of the two landscapes (Dvir and Lachish), the three dependent variables (spider alpha, beta and gamma diversities), and the seven patch-cardinalities (3-9 patches) for a total of 42 
analyses. In each analysis we used the information from all relevant pseudo-landscapes and first fitted a multiple linear regression model of the dependent variable against all 11 explanatory variables. We then explored the relative importance of each variable using the LMG method (R package 'relaimpo') that can deal with correlated variables and returns independent relative importance values, which can be summed over variables according to scale or thematic group (Groemping 2006). The summation property is extremely important here, as it allows aggregating the relative importance values by thematic group or by scale. To ensure comparability of the variance partitioning among the 42 analyses we maintained all of the explanatory variables in all multiple linear regression analyses.

To explore whether the variance partitioning results can arise by chance, we permutated the values of the dependent variable (spiders' alpha, beta or gamma) 100 times for each of the 42 analyses, and for each permutation we re-run the multiple linear regression and LMG analyses. We then calculated the mean explained variance over the 100 permutations and compared it to the explained variance of the original, non-permutated dataset.

In addition, focusing only on the six scale-specific prey and habitat variables, for each analysis we calculated the ratio between the mean explained variance per scale-matching variable and the mean explained variance per cross-scale variable. For example, for the models with spiders' beta diversity as the dependent variable, we took the mean of the explained variance by preys' beta and habitats' beta for scale-matching. We took the mean of preys' alpha, preys' gamma, habitats' alpha and habitats' gamma for cross-scale. We took the ratio of the two, with values above 1 indicating the scale-matching explaining more per variable than cross-scale.

Finally, to focus exclusively on scale matching, we fitted for each dependent variable (in each landscape and patch cardinality) a set of 3 multiple regression models, the first with preys' alpha and habitats' alpha as explanatory variables, the second with preys' beta and habitats' beta as explanatory variables and the last with preys' gamma and habitats' gamma as explanatory variables. For each model we calculated the total explained variance. We compared the models performance using Akike Information Criteria corrected for small sample size (AICc). If scale matching is more important than cross-scale we expect the scale-matching model to have the highest explained variance and the smallest AICc.

All analyses were done in $\mathrm{R}$ statistical platform ( $\mathrm{R}$ Core Team 2016). We report in the main text only the results for 9 patches, yet the results for all patch cardinalities can be found in Online Resource 2.

\section{Results}

\section{General results}

The numbers of spider species and prey groups did not differ much between Lachish and Dvir, yet differences in total abundances were observed. In Dvir we sampled a total of 2026 spider individuals from 114 species or morphospecies, along with an additional 6274 other invertebrates, representing 19 different orders or families. In Lachish, we sampled 3742 spider individuals from 115 species and 5875 other invertebrates from 18 families or orders. Online Resource 1 in the supporting information contains the area and sampling intensity in each patch, the relative cover of each habitat, and the abundance in each patch of each spider species or prey group. 
Spider mean alpha diversity did not change much with the number of patches (Online Resource 2), but it was higher in Dvir than in Lachish for all patch-cardinalities. Both beta and gamma diversity increased with the number of patches in both landscapes. Similar to alpha diversity, gamma diversity was also consistently lower in Lachish than in Dvir (Online Resource 2, Fig. S1).

\section{Alpha, beta and gamma diversity change with drivers}

In both landscapes, spider alpha, beta and gamma diversities were correlated with explanatory variables from all five thematic groups: habitat diversity (Fig. 1), prey diversity (Fig. 2), area, connectivity, and sampling (Online Resource 2).

In both landscapes, there were positive correlations between species diversity components and the scale-corresponding habitat diversity components, as predicted by the

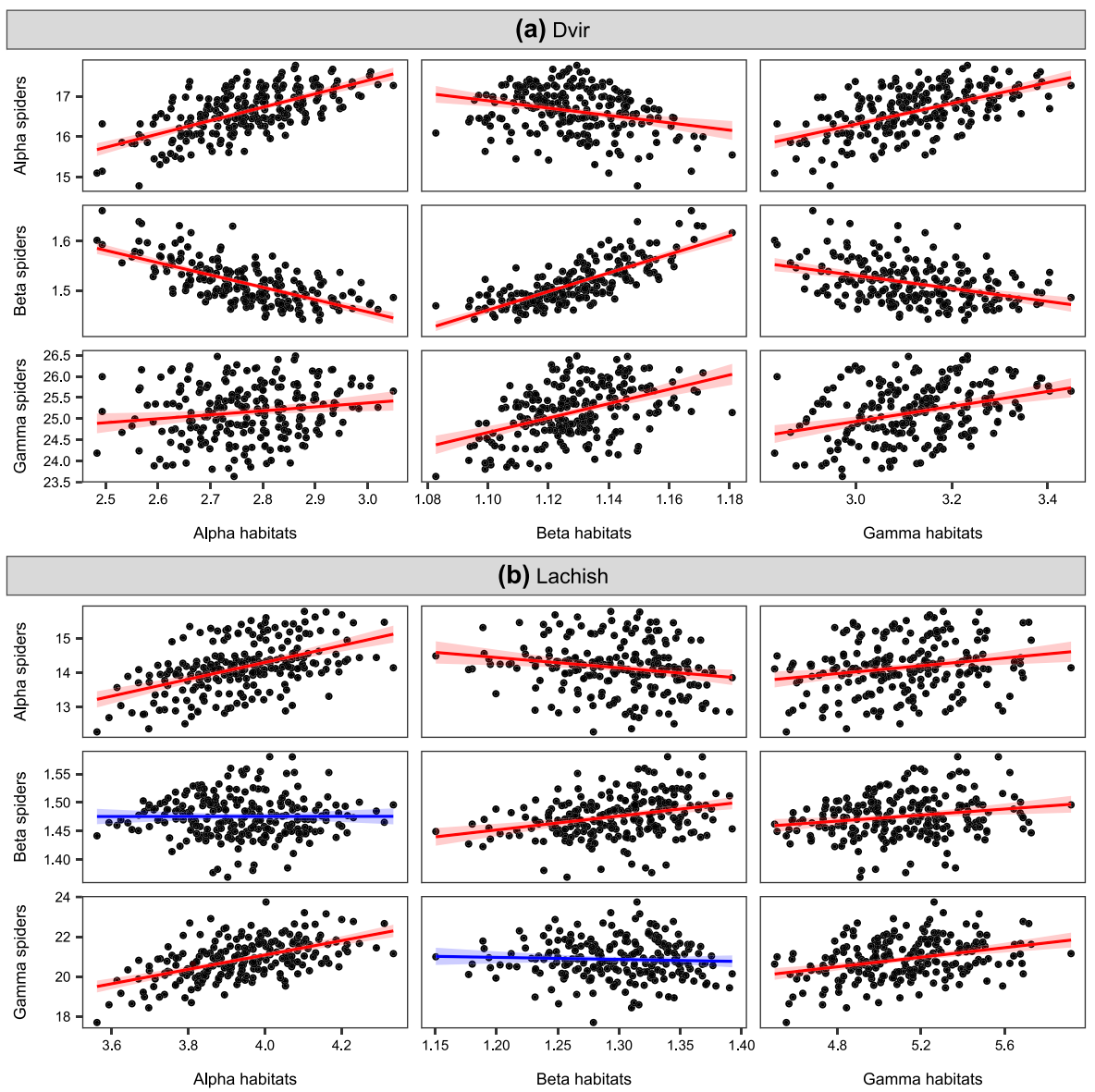

Fig. 1 The change of spider alpha, beta and gamma diversities with habitat alpha, beta and gamma diversities, in each of the landscapes. Solid lines are linear models (with 0.95 confidence intervals), red lines are statistically significant $(\mathrm{P} \leq 0.5)$, and blue lines are non-significant. (Color figure online) 


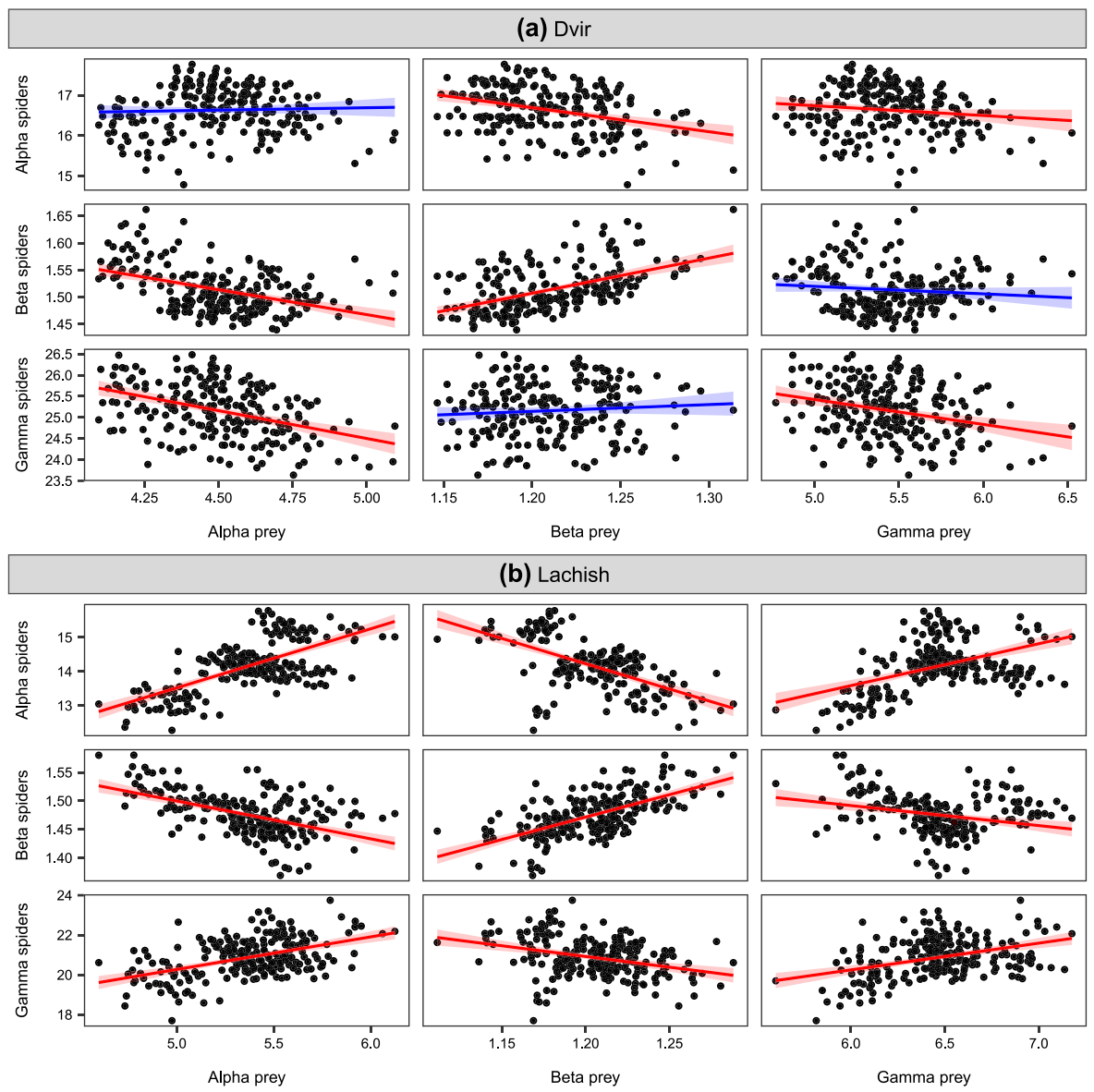

Fig. 2 The change of spider alpha, beta and gamma diversities with prey alpha, beta and gamma diversities, in each of the landscapes. Solid lines are linear models (with 0.95 confidence intervals), red lines are statistically significant $(\mathrm{P} \leq 0.5)$, and blue lines are non-significant. (Color figure online)

scale-matching hypothesis. That is, spiders' alpha diversity increased with habitat alpha diversity, spiders' beta diversity increased with habitat beta diversity and spiders' gamma diversity increased with habitat gamma diversity (Fig. 1). However, we also found considerable cross-scale effects in both landscapes. In some cases, the cross-scale effects were positive (e.g., spiders' alpha diversity increased with habitat gamma diversity and spiders' gamma diversity increased with habitat alpha diversity, Fig. 1). In others, they were negative (e.g., spiders' alpha diversity decreased with habitat beta diversity).

The response of spider diversity to prey diversity differed among the two landscapes. In Lachish we found the expected positive trends of scale matching (Fig. 2b). We also found both positive and negative cross-scales responses. However, in Dvir, where prey diversity contributed very little to the overall explained variance (Fig. 3), scale matching was observed only for beta diversity (Fig. 2a). Instead, spider alpha diversity did not vary significantly with prey alpha diversity, while spider gamma diversity actually decreased with prey gamma diversity. 

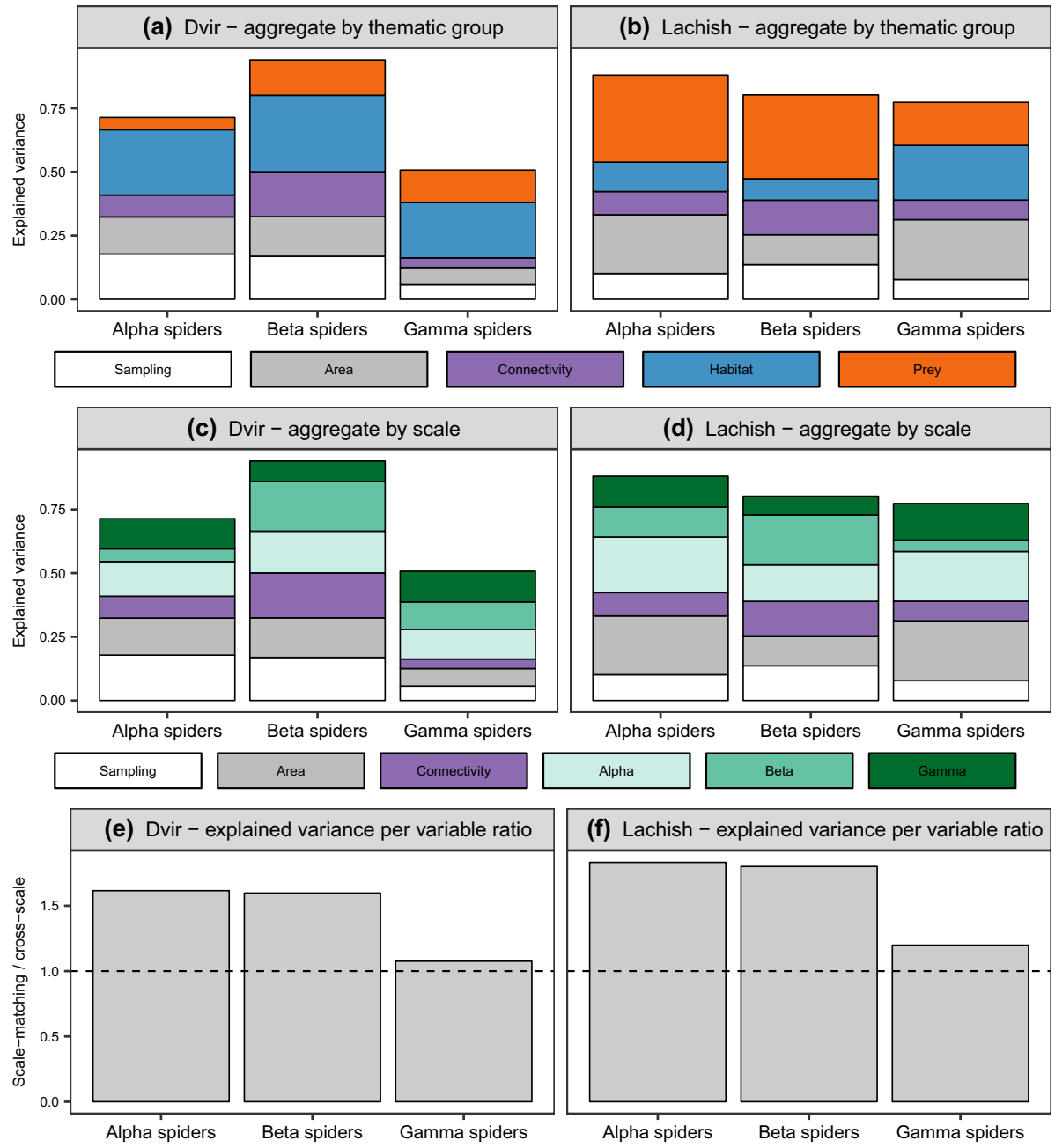

Fig. 3 The results of the variance partitioning analysis for spider alpha, beta and gamma diversity in Dvir $(\mathbf{a}, \mathbf{c}, \mathbf{e})$ and Lachish $(\mathbf{b}, \mathbf{d}, \mathbf{f})$. a, b The explained variance when the 11 explanatory variables are aggregated by the thematic group. c, d The explained variance when the prey/habitat variables are aggregated according to scale (and the remaining variables according to thematic group). $\mathbf{e}, \mathbf{f}$ The ratio between the mean explained variance per scale-matching variable and the mean explained variance per cross-scale variable (for prey and habitat variables)

Spider alpha, beta and gamma diversities also responded to variation in the explanatory variables from the area, connectivity and sampling thematic groups (Online Resource 2, Figures S4 and S5). In general, the responses of spider alpha and gamma diversities to various explanatory variables followed a similar trend whereas the response of spider beta diversity was of the opposite direction. For example, spider alpha and spider gamma increased with the total area, the clumping index and the total number of samples. By contrast, spider beta diversity decreased with the same explanatory variables. Patterns in relation to area evenness and minimum spanning tree were 
more complex. These results potentially reflect beta being the gamma to alpha ratio in a multiplicative diversity partitioning framework (e.g., if both alpha and gamma increase in relation to a certain variable, but alpha is increasing faster than gamma, beta would show a decreasing trend).

\section{Variance partitioning}

The total explained variance of spiders alpha, beta or gamma diversity in the LMG analyses ranged between 0.393 and 0.940 , with a mean of 0.698 ( \pm 0.023 SE). However, when permuting the dependent variables, the total explained variance was considerably lower and ranged between 0.011 and 0.052 , with a mean $( \pm S E)$ of $0.026 \pm 0.002$ (Online Resource 3), suggesting that the observed relation between the explanatory and dependent variables are unlikely to arise by chance alone. The patterns reported below were consistent in relation to the number of patches (online resources $2,4,5$ ).

In Dvir, spider alpha diversity was mostly affected by sampling effort, the total area and the alpha component of habitat diversity, while all components of prey diversity contributed very little (Table 1). Spider beta diversity was mainly explained by the clumping index, the beta components of habitat and by sampling and total area. The overall explained variance of spider gamma diversity was lower than that for alpha and beta diversities, yet the explained variance was more evenly distributed among the different explanatory variables, scales, and thematic groups.

In Lachish, spider alpha diversity was mainly explained by prey alpha, along with the sampling and area variables, and the other scaling components of prey diversity (Table 1). Spider beta diversity was mainly affected by prey beta diversity, the total number of samples and the minimum spanning tree. For spider gamma diversity, alpha habitat and the area evenness were the most important variables.

The two landscapes differed considerably in the overall contribution of variables from the 5 thematic groups. The two landscapes differed in the relative contributions of habitat and prey, with the first being dominant in Dvir and the second in Lachish (Fig. 3a,b). In addition, the area variables were more important in Lachish, while the sampling variables explained more variance in Dvir.

We found considerable support for the scale-matching hypothesis. Overall, in both landscapes the combined contribution of the variables that exhibited scale-matching exceeded the variance explained by cross-scale diversity components in $81 \%$ of cases ( 34 out of 42 variance partitioning analyses). In 23 out of 42 analyses the scale-matching component was the most important among the six compared groups (sampling, area, connectivity, alpha, beta and gamma). In both landscapes, most of the support for scale matching was found for spider alpha and spider beta. First, for alpha and beta the variable that explained the most variance from the 6 prey and habitat variables was the scale matching one (see bold in Table 1). Second, for all but gamma spiders in Lachish, the matching scale component explained more variance than any of the cross scale component (Fig. 3c,d). Third, the ratio between the mean explained variance per scale matching variable and the mean explained variance per cross-scale variable was larger than one in all cases (Fig. 3e,f). For spiders alpha and beta diversities, the ratios ranged from 1.60 to 1.83 , suggesting that scale matching variable explained at least $60 \%$ more variation than cross scale variable, while for spiders gamma diversity the ratio was only slightly larger than one. 
Table 1 Results for the LMG analysis with 9 patches for Dvir and Lachish for each dependent variable (spider's alpha, beta or gamma). (Color figure online)

\begin{tabular}{|c|c|c|c|c|c|c|}
\hline \multirow{2}{*}{$\begin{array}{l}\text { Landscape } \\
\text { Dependent (spider) }\end{array}$} & \multicolumn{3}{|c|}{ Dvir } & \multicolumn{3}{|c|}{ Lachish } \\
\hline & Alpha & Beta & Gamma & Alpha & Beta & Gamma \\
\hline F value & 47.21 & 294.44 & 19.46 & 139.00 & 76.70 & 64.54 \\
\hline$p$ value & $<0.001$ & $<0.001$ & $<0.001$ & $<0.001$ & $<0.001$ & $<0.001$ \\
\hline \multicolumn{7}{|l|}{ Explained variance } \\
\hline Total number of samples & 0.18 & 0.17 & 0.06 & 0.10 & 0.14 & 0.08 \\
\hline Total area & 0.12 & 0.14 & 0.05 & 0.10 & 0.10 & 0.09 \\
\hline Area evenness & 0.02 & 0.01 & 0.01 & 0.13 & 0.02 & 0.14 \\
\hline Minimum spanning tree & 0.01 & 0.01 & 0.02 & 0.01 & 0.11 & 0.02 \\
\hline Clumping index & 0.08 & 0.17 & 0.02 & 0.08 & 0.03 & 0.06 \\
\hline Alpha habitat & 0.13 & 0.11 & 0.05 & 0.06 & 0.03 & 0.11 \\
\hline Beta habitat & 0.03 & 0.14 & 0.09 & 0.02 & 0.02 & 0.02 \\
\hline Gamma habitat & 0.11 & 0.06 & 0.08 & 0.03 & 0.03 & 0.08 \\
\hline Alpha prey & 0.01 & 0.06 & 0.07 & 0.15 & 0.11 & 0.08 \\
\hline Beta prey & 0.02 & 0.06 & 0.02 & 0.10 & 0.17 & 0.02 \\
\hline Gamma prey & 0.01 & 0.02 & 0.04 & 0.09 & 0.04 & 0.06 \\
\hline Total & 0.71 & 0.94 & 0.51 & 0.88 & 0.80 & 0.77 \\
\hline $\mathrm{R}^{2}$ in permutations & 0.05 & 0.05 & 0.05 & 0.05 & 0.05 & 0.05 \\
\hline
\end{tabular}

The partitioning of the total explained variance to each variable is shown. Gray filled cells are those that contribute to scale-matching, blue filled cells for cross-scale, while empty cells do not correspond to any specific scale. The variable with the largest explained variance among the six prey/habitat variables is given in bold

\section{Model selection analysis}

The LMG results are mirrored in the model-selection analyses, where the scale-matching model explained more variance than any of the cross-scale models for spider alpha and beta (Fig. 4, left and middle columns). However, for spider gamma diversity, a cross-scale model of alpha diversity was selected over the scale-matching model (Fig. 4, right column). This is also evident in the scale-matching models receiving an AICc weight of 1 for spider alpha and beta, while cross-scale alpha model receiving high AICc weights for spider gamma. 


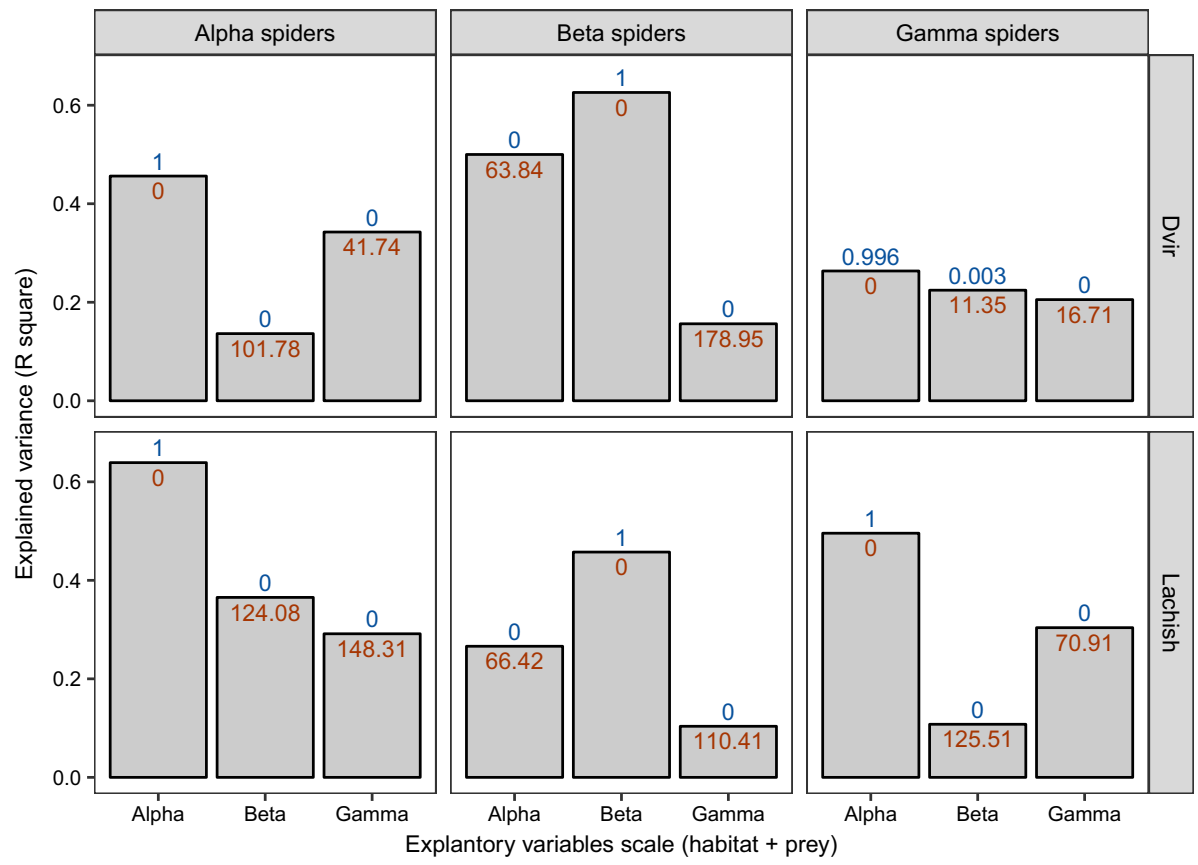

Fig. 4 The results from the model-selection analysis for spiders alpha, beta and gamma diversity in each landscape. Three models where fitted in each panel, each with the habitat and prey variables at the alpha, beta or gamma scale. Bars are the explained variance, red text below bar lines are the delta AICc values and blue text above bar lines are the AICc weights. (Color figure online)

\section{Discussion}

The main aim of this paper is to test the scale-matching hypothesis using spider diversity and a set of 11 explanatory variables from five thematic groups (sampling, area, connectivity, habitat diversity and prey diversity) in two fragmented agricultural landscapes. In general, we found considerable evidence for the scale-matching hypothesis, with alpha, beta and (less so) gamma diversities of spiders being mainly explained by their respective prey and/or habitat diversities (Table 1; Figs. 3, 4). We further found a strong effect of connectivity on spider beta diversity, but not on alpha and gamma diversities (Table 1; Fig. 3).

We found a noticeable difference between Dvir and Lachish in the relative importance of the different thematic groups, with habitat variables being more important in Dvir (Fig. 3a) and prey variables being more important in Lachish (Fig. 3b). It is possible that the larger number of prey individuals per spider in Dvir $(6274 / 2026=3.10$ prey individuals per spider individual) versus Lachish $(5875 / 3742=1.57)$ results in stronger competition for prey in Lachish. Also, spider guild structure (sensu: Cardoso et al. 2011) differs between the two landscapes with web building spider taking a larger proportion of the total abundance in Lachish than in Dvir (20.4\% vs. $7.6 \%$, respectively). Although spiders are generally considered generalist predators, they may exhibit high levels of specificity at the taxonomic resolution we applied here. If so, web building spiders may be more specific in the groups of prey they target, causing a stronger effect of prey diversity on spider diversity. Alternatively, the two landscapes differ in their alpha and gamma habitat diversities (Fig. 1), with 
relatively low values in Dvir (alpha: 2.48-3.05, gamma: 2.83-3.45, Fig. 1) and high values in Lachish (alpha: 3.56-4.34, gamma: 4.5-5.92). These differences do not reflect a considerable change in the proportional cover of perennials, but rather a high representation of exposed soil in Dvir in comparison to high proportion of annuals and thistles in Lachish. In other words, the within-patch matrix is generally more hostile in Dvir than in Lachish, providing less cover from predation by reptiles and birds. Spider species that are more sensitive to predation survive mostly in patches with higher habitat diversity (safer within-patch matrix), resulting with a strong correlation between spider and habitat diversities.

Both habitat (Heikkinen and MacMahon 2004; Robinson 1981; Rypstra et al. 1999; Uetz 1991) and prey (Nentwig 1986; Sanders et al. 2015) may be considered as resources for spiders, and can thus be considered under a niche-related framework. If niche processes are dominant in structuring the spider community, we expect to find strong evidence of scalematching. Indeed, despite the differences between landscapes in the relative importance of prey and habitat, patterns of scale matching were similar. We found evidence of scale matching in the linear regression analyses of either habitat (both landscapes, Fig. 1a,b) or prey (Lachish, Fig. 2b). In the variance partitioning analyses we observed scale-matching for spiders' alpha and beta (Fig. 3c,d), with scale-matching variable explaining 1.60 to 1.83 times as much of the variance in spider diversity than a cross-scale variable (Fig. 3e,f). Finally, the model selection analyses revealed that the scale-matching model was the best model for both alpha and beta diversity of spiders in both landscapes (Fig. 4).

On the contrary, in both landscape we found limited support in the variance portioning and model selection analyses for scale-matching at the spider gamma scale. In the variance partitioning alpha variables explained more variance than gamma in Lachish, and roughly the same amount in Dvir (Fig. 3c,d). Although the mean explained variance value per scale-matching variable was larger than that for cross-scale (Fig. 3e,f), the actual values were very similar. The model selection analysis revealed that spider gamma diversity is better explained by alpha diversity of prey and habitat than by any other scale components (Fig. 4). The cross-scale effects may arise from dependency between the different diversity components. Under fixed gamma diversity, higher alpha diversity is usually accompanied by a lower beta diversity, despite the mathematical independence of the alpha and beta under multiplicative partitioning (Baselga 2010). Indeed, we observed opposite trends of alpha and beta against many explanatory variables. In addition, increasing alpha diversity of resources may allow more species to sustain viable populations in each patch. This increase in species occupancy level (number of patches in which the species is found), may translate to higher gamma diversity, since species meta-populations are less susceptible to extinction. In Dvir, we also observed a relatively strong effect of habitat gamma diversity on spider alpha diversity (Fig. 3c). Increasing the habitat gamma diversity may results in different patches supporting large populations of different species. Then, inter-patch dispersal of individuals (e.g., mass-effect dynamics) may allow species whose preferred habitat is not found in a certain patch to sustain a population there through immigration from nearby patches where their preferred habitat is found (Leibold et al. 2004; Mouquet and Loreau 2002; Shmida and Wilson 1985).

Dispersal limitation is expected to increase beta diversity (Kneitel and Chase 2004). Indirectly, increased dispersal limitation may decrease gamma and alpha diversities as well. Alpha diversity may decline because of decreased colonization rates and reduced rescue effects (Brown and Kodric-Brown 1977; Kneitel and Chase 2004; Leibold et al. 2004; Mouquet and Loreau 2002). In our study, we estimated connectivity with two gamma-scale variables, the minimum spanning tree (larger values mean less connectivity and greater dispersal limitation) and the clumping index (larger values mean more connectivity and 
smaller dispersal limitation). As expected, in both landscapes, connectivity had a crossscale negative effect on spider beta diversity, and a much weaker positive effect on alpha and gamma. However, the landscapes differ in the contribution of specific connectivity measures to the beta-diversity-connectivity relationship. In Dvir we observed a strong negative trend of spider beta diversity with the clumping index (online resource 2), whereas in Lachish we observed a strong positive trends of spider beta diversity with the increasing length of the minimum spanning tree. The variance partitioning analysis further strengthen these results, given that clumping was more important in Dvir and the spanning tree was more important in Lachish (Table 1). Furthermore, connectivity mainly affected spider beta diversity and contributed little explained variance to spider alpha and gamma diversities (Fig. 3).

In a more methodological approach, Zhang et al. (2014) correctly claimed that exploring the dependency of alpha, beta and gamma diversities on multiple environmental and spatial variables requires multiple landscapes, as each landscape returns a single value for each diversity component. Here we have used information from two landscapes to create two sets of multiple 'pseudo-landscapes'. Clearly, one cannot claim that the pseudo-landscapes are truly independent from one another or that the sub-sampling increased the actual number of replicates in our study. At the end, our conclusions are restricted to the two landscapes we had data for. However, scale-matching is a simple and fundamental hypothesis, which remained largely unexplored. In fact, many studies implicitly assume scale-matching by selecting explanatory variables to be included in the analysis and the scale at which they are measured. In our study, we examine this assumption in a simple two-landscape system and present an approach of partitioning drivers that can be generally used to test the scalematching hypothesis in greater details in multiple-landscape cases.

Within the limits of our analysis we found agreement with the scale matching hypothesis, mainly at the alpha and beta scales, as well as evidence for cross-scale effects at the gamma scale. We further found that including variables from multiple thematic groups and multiple scales is required if we wish to explain diversity patterns. The drop in explained variance when randomizing the scale components of spider diversities suggests that the relations we have found are unlikely to arise by chance. If we are to better understand the spatial distribution of species diversity, we need to better understand the scaling properties of diversity. When doing so we need to remember that scaling of diversity is not limited only to species, but relates to various other aspects such as the distribution in space and time of patches, resources and habitats and to the way we choose to quantify them. Moreover, we believe scale-matching is a fundamental hypothesis in ecology, which we too often tend to take for granted. We hope that exploring multiple scale components of both diversity and drivers would increase our understating of diversity pattern and our ability to conserve them.

Acknowledgements This study was supported by the Israel Science Foundation (Grant 751/09) to YZ. We thank Richard M. Gunton for his helpful review and constructive comments. This is publication \#1003 of the Mitrani Department of Desert Ecology.

Author contributions YG collected the data. YG and IG conceived the idea, designed the study and analysed the data. YG wrote the first draft of the manuscript. All authors contributed considerably to later version of the manuscripts.

Data availability All data generated or analyzed during this study are included in this published article [and supplementary information files]. 
OpenAccess This article is distributed under the terms of the Creative Commons Attribution 4.0 International License (http://creativecommons.org/licenses/by/4.0/), which permits unrestricted use, distribution, and reproduction in any medium, provided you give appropriate credit to the original author(s) and the source, provide a link to the Creative Commons license, and indicate if changes were made.

\section{References}

Baselga A (2010) Multiplicative partition of true diversity yields independent alpha and beta components; additive partition does not. Ecology 91:1974-2112. https://doi.org/10.1890/09-0320.1

Brown JH, Kodric-Brown A (1977) turnover rates in insular biogeography-effect of immigration and extinction. Ecology 58:445-449. https://doi.org/10.2307/1935620

Cardoso P, Pekár S, Jocqué R, Coddington JA (2011) Global patterns of guild composition and functional diversity of spiders. PLoS ONE 6:e21710. https://doi.org/10.1371/journal.pone.0021710

Crist TO, Veech JA (2006) Additive partitioning of rarefaction curves and species-area relationships: unifying alpha-, beta- and gamma-diversity with sample size and habitat area. Ecol Lett 9:923-932. https://doi.org/10.1111/j.1461-0248.2006.00941.x

Ekroos J, Heliölä J, Kuussaari M (2010) Homogenization of lepidopteran communities in intensively cultivated agricultural landscapes. J Appl Ecol 47:459-467. https://doi.org/10.111 1/j.1365-2664.2009.01767.x

Fahrig L (2013) Rethinking patch size and isolation effects: the habitat amount hypothesis. J Biogeogr 40:1649-1663. https://doi.org/10.1111/jbi.12130

Fournier B, Mouly A, Moretti M, Gillet F (2017) Contrasting processes drive alpha and beta taxonomic, functional and phylogenetic diversity of orthopteran communities in grasslands. Agric Ecosyst Environ 242:43-52. https://doi.org/10.1016/j.agee.2017.03.021

Gavish Y, Ziv Y (2016) Joint effect of habitat identity and spatial distance on spiders' community similarity in a fragmented transition zone. PLoS ONE 11:e0168417. https://doi.org/10.1371/journ al.pone.0168417

Gavish Y, Ziv Y, Rosenzweig ML (2012) Decoupling fragmentation from habitat loss for spiders in patchy agricultural landscapes. Conserv Biol 26:150-159

Giladi I, Ziv Y, May F, Jeltsch F (2011) Scale-dependent determinants of plant species richness in a semi-arid fragmented agro-ecosystem. J Veg Sci 22:983-996. https://doi.org/10.111 1/j.1654-1103.2011.01309.x

Gossner MM et al (2016) Land-use intensification causes multitrophic homogenization of grassland communities. Nature 540:266. https://doi.org/10.1038/nature20575

Groemping U (2006) Relative Importance for linear regression in R: the package relaimpo. J Stat Softw 17:1-27

Hanski I, Zurita GA, Bellocq MI, Rybicki J (2013) Species-fragmented area relationship. Proc Natl Acad Sci. https://doi.org/10.1073/pnas.1311491110

Heikkinen MW, MacMahon JA (2004) Assemblages of spiders on models of semi-arid shrubs. J Arachnol 32:313-323

Hendrickx F et al (2009) Pervasive effects of dispersal limitation on within- and among-community species richness in agricultural landscapes. Glob Ecol Biogeogr 18:607-616. https://doi.org/10.111 1/j.1466-8238.2009.00473.x

Hortal J, Borges PAV, Gaspar C (2006) Evaluating the performance of species richness estimators: sensitivity to sample grain size. J Anim Ecol 75:274-287

Hortal J, Triantis KA, Meiri S, Thebault E, Sfenthourakis S (2009) Island species richness increases with habitat diversity. Am Nat 174:E205-E217. https://doi.org/10.1086/645085

Hubbell SP (2001) The unified neutral theory of biodiversity and biogeography. Princeton University Press, Princeton, New Jersey

Jamoneau A, Chabrerie O, Closset-Kopp D, Decocq G (2012) Fragmentation alters beta-diversity patterns of habitat specialists within forest metacommunities. Ecography 35:124-133. https://doi.org/1 0.1111/j.1600-0587.2011.06900.x

Jost L (2007) Partitioning diversity into independent alpha and beta components. Ecology 88:24272439. https://doi.org/10.1890/06-1736.1

Jost L (2010) Independence of alpha and beta diversities. Ecology 91:1969-2104. https://doi. org/10.1890/09-0368.1 
Kemp JE, Ellis AG (2017) Significant local-scale plant-insect species richness relationship independent of abiotic effects in the temperate cape floristic region biodiversity hotspot. PLoS ONE 12:16. https ://doi.org/10.1371/journal.pone.0168033

Kneitel JM, Chase JM (2004) Trade-offs in community ecology: linking spatial scales and species coexistence. Ecol Lett 7:69-80

Lande R (1996) Statistics and partitioning of species diversity, and similarity among multiple communities. Oikos 76:5-13. https://doi.org/10.2307/3545743

Leibold MA et al (2004) The metacommunity concept: a framework for multi-scale community ecology. Ecol Lett 7:601-613

Malavasi M, Conti L, Carboni M, Cutini M, Acosta ATR (2016) Multifaceted analysis of patch-level plant diversity in response to landscape spatial pattern and history on mediterranean dunes. Ecosystems 19:850-864. https://doi.org/10.1007/s10021-016-9971-4

Martinez E, Ros M, Bonilla MA, Dirzo R (2015) Habitat heterogeneity affects plant and arthropod species diversity and turnover in traditional cornfields. PLoS ONE 10:19. https://doi.org/10.1371/journ al.pone.0128950

Mendenhall CD, Karp DS, Meyer CFJ, Hadly EA, Daily GC (2014) Predicting biodiversity change and averting collapse in agricultural landscapes. Nature 509:213-217. https://doi.org/10.1038/nature13139

Mouquet N, Loreau M (2002) Coexistence in metacommunities: the regional similarity hypothesis. Am Nat 159:420-426. https://doi.org/10.1086/338996

Nentwig W (1986) Non-webbuilding spiders: prey specialists or generalists? Oecologia 69:571-576. https:// doi.org/10.2307/4217987

Papanikolaou AD et al (2017) Wild bee and floral diversity co-vary in response to the direct and indirect impacts of land use. Ecosphere 8:e02008. https://doi.org/10.1002/ecs2.2008

Poggio SL, Chaneton EJ, Ghersa CM (2010) Landscape complexity differentially affects alpha, beta, and gamma diversities of plants occurring in fencerows and crop fields. Biol Conserv 143:2477-2486. https://doi.org/10.1016/j.biocon.2010.06.014

Prober SM et al (2015) Plant diversity predicts beta but not alpha diversity of soil microbes across grasslands worldwide. Ecol Lett 18:85-95. https://doi.org/10.1111/ele.12381

R Core Team (2016) R: a language and environment for statistical computing. R Foundation for Statistical Computing, Vienna, Austria. http://www.R-projectorg

Robinson JV (1981) The effect of architectural variation in habitat on a spider community-an experimental field-study. Ecology 62:73-80

Rosenzweig ML (1995) Species diversity in space and time. Cambridge University Press, Cambridge

Rypstra AL, Carter PE, Balfour RA, Marshall SD (1999) Architectural features of agricultural habitats and their impact on the spider inhabitants. J Arachnol 27:371-377

Sanders D, Vogel E, Knop E (2015) Individual and species-specific traits explain niche size and functional role in spiders as generalist predators. J Anim Ecol 84:134-142. https://doi. org/10.1111/1365-2656.12271

Sandom C et al (2013) Mammal predator and prey species richness are strongly linked at macroscales. Ecology 94:1112-1122. https://doi.org/10.1890/12-1342.1

Shmida A, Wilson MV (1985) Biological determinants of species-diversity. J Biogeogr 12:1-20. https://doi. org/10.2307/2845026

Simmering D, Waldhardt R, Otte A (2006) Quantifying determinants contributing to plant species richness in mosaic landscapes: a single- and multi-patch perspective. Landscape Ecol 21:1233-1251. https:// doi.org/10.1007/s10980-006-0027-6

Stewart AJA, Wright AF (1995) A new inexpensive suction apparatus for sampling arthropods in grassland. Ecol Entomol 20:98-102

Tews J, Brose U, Grimm V, Tielborger K, Wichmann MC, Schwager M, Jeltsch F (2004) Animal species diversity driven by habitat heterogeneity/diversity: the importance of keystone structures. J Biogeogr 31:79-92

Triantis KA, Guilhaumon F, Whittaker RJ (2012) The island species-area relationship: biology and statistics. J Biogeogr 39:215-231. https://doi.org/10.1111/j.1365-2699.2011.02652.x

Uetz GW (1991) Habitat structure and spider foraging. In: Bell SS, McCoy ED, Mushinsky HR (eds) Habitat structure-the physical arrangement of objects in space. Chapman and Hall, London, pp 325-348

Urban D, Keitt T (2001) Landscape connectivity: a graph-theoretic perspective. Ecology 82:1205-1218. https://doi.org/10.1890/0012-9658(2001)082\%5b1205:lcagtp\%5d2.0.co;2

Weibull AC, Ostman O, Granqvist A (2003) Species richness in agroecosystems: the effect of landscape, habitat and farm management. Biodivers Conserv 12:1335-1355. https://doi.org/10.1023/a:10236 17117780

Whittaker RH (1972) Evolution and measurement of species diversity. Taxon 21:213-251 
Wulf M, Kolk J (2014) Plant species richness of very small forests related to patch configuration, quality, heterogeneity and history. J Veg Sci 25:1267-1277. https://doi.org/10.1111/jvs.12172

Yang JW, Wu WX, Chung CC, Chiang KP, Gong GC, Hsieh CH (2018) Predator and prey biodiversity relationship and its consequences on marine ecosystem functioning-interplay between nanoflagellates and bacterioplankton. ISME J 12:1532-1542. https://doi.org/10.1038/s41396-018-0111-3

Zhang J, Crist TO, Hou P (2014) Partitioning of $\alpha$ and $\beta$ diversity using hierarchical Bayesian modeling of species distribution and abundance. Environ Ecol Stat 21:611-625. https://doi.org/10.1007/s1065 $1-013-0271-2$

Zulka KP et al (2014) Species richness in dry grassland patches of eastern Austria: a multi-taxon study on the role of local, landscape and habitat quality variables. Agr Ecosyst Environ 182:25-36. https://doi. org/10.1016/j.agee.2013.11.016

\section{Affiliations}

\section{Yoni Gavish ${ }^{1,2}$ (D) Itamar Giladi ${ }^{3}(\mathbb{D}) \cdot$ Yaron Ziv $^{2}$}

Itamar Giladi

itushgi@bgu.ac.il

Yaron Ziv

yziv@bgu.ac.il

1 School of Biology, Faculty of Biological Sciences, University of Leeds, Leeds LS29JT, UK

2 Spatial Ecology Lab, Department of Life Sciences, Ben-Gurion University of the Negev, 8410501 Beer Sheva, Israel

3 Mitrani Department of Desert Ecology, Swiss Institute for Dryland Environmental and Energy Research, Jacob Blaustein Institutes for Desert Research, Ben-Gurion University of the Negev, Sede Boqer Campus, 8499000 Sede Boqer, Israel 